\title{
Propriedades Nutritivas e Bioativas do Araçá (Psidium cattleianum Sabine)
}

\author{
Nutritive and Bioactive Properties of Araçá (Psidium cattleianum Sabine) \\ Propriétés Nutritives et Bioactives de L'araç (Psidium cattleianum Sabine)
}

Recebido: 06/01/2022 | Revisado: 11/01/2022 | Aceito: 14/01/2022 | Publicado: 16/01/2022

Eloir Pereira Gwozdz

ORCID: https://orcid.org/0000-0002-6418-334X Universidade Regional Integrada do Alto Uruguai e das Missões, Brasil E-mail: nutrielopereira@gmail.com

Marília Jordana Dequi Vendrúsculo

ORCID: https://orcid.org/0000-0002-8154-4143 Universidade Regional Integrada do Alto Uruguai e das Missões, Brasil E-mail: maajordanaa@gmail.com

Luiz Henrique Menosso

ORCID: https://orcid.org/0000-0001-7862-2254 Universidade Regional Integrada do Alto Uruguai e das Missões, Brasil E-mail: luizhenriquemenosso@gmail.com

Heloísa Chaves Tasca

ORCID: https://orcid.org/0000-0002-7067-1411 Universidade Regional Integrada do Alto Uruguai e das Missões, Brasil E-mail: helochavestasca@hotmail.com

Yasmim Miryan Machado

ORCID: https://orcid.org/0000-0002-3025-4824

Universidade Regional Integrada do Alto Uruguai e das Missões, Brasil E-mail: yasmimmachado273@gmail.com

Victor Alberto Stachelski

ORCID: https://orcid.org/0000-0001-7337-946X Universidade Regional Integrada do Alto Uruguai e das Missões, Brasil

E-mail: victoralbertostachelski@gmail.com

Sabrina Pavan Zannoni

ORCID: https://orcid.org/0000-0001-9119-5867

Universidade Regional Integrada do Alto Uruguai e das Missões, Brasil

E-mail: sabrinazannoni171710@gmail.com

Marcieli Peruzzolo

ORCID: https://orcid.org/0000-0001-9012-2199

Universidade Regional Integrada do Alto Uruguai e das Missões, Brasil

E-mail: marciperuzzolo@yahoo.com.br

Giovana Cristina Ceni

ORCID: https://orcid.org/0000-0003-2787-7593

Universidade Federal de Santa Maria, Brasil

E-mail: giovana.ceni@ufsm.br

Rosicler Colet

ORCID: https://orcid.org/0000-0001-8589-0804 Universidade Regional Integrada do Alto Uruguai e das Missões, Brasil E-mail: rosicler.colet@yahoo.com.br

Jamile Zeni

ORCID: https://orcid.org/0000-0002-0529-2212 Universidade Regional Integrada do Alto Uruguai e das Missões, Brasil

E-mail: jamilezeni@uricer.edu.br

Geciane Toniazzo Backes

ORCID: https://orcid.org/0000-0001-8652-8399

Universidade Regional Integrada do Alto Uruguai e das Missões, Brasil

E-mail: gtoniazzo@uricer.edu.br

\section{Resumo}

Os consumidores estão cada vez mais em busca de alimentos que possuam compostos benéficos à saúde, também chamados de bioativos ou fotoquímicos, os quais são substâncias derivadas do metabolismo secundário dos vegetais. O Brasil possui uma das maiores biodiversidades do mundo, com fontes inexploradas de frutas nativas com altos teores de compostos bioativos. Dentre essas frutas, se destaca o araçá (Psidium cattleianum Sabine). O araçá, além do alto valor nutricional, possui quantidades significativas de compostos fenólicos, sendo epicatequina e ácido gálico seus componentes principais, ácidos graxos insaturados e carotenoides. Por apresentar estas características, está sendo considerada uma planta promissora para a indústria farmacêutica e alimentícia devido ao seu potencial de aplicação 
como alimento fitoterápico, funcional, entre outros. Neste sentido, este trabalho tem como objetivo apresentar uma revisão bibliográfica sobre o araçá e seus benefícios.

Palavras-chave: Araçá-vermelho; Araçá-amarelo; Antioxidantes; Aplicação.

\begin{abstract}
Consumers are increasingly looking for foods that have compounds that are beneficial to health, also called bioactive or photochemical, which are substances derived from the secondary metabolism of vegetables. Brazil has one of the greatest biodiversity in the world, with unexplored sources of native fruits with high levels of bioactive compounds. Among these fruits, the araçá (Psidium cattleianum Sabine) stands out. Araçá, in addition to its high nutritional value, has significant amounts of phenolic compounds, with epicatechin and gallic acid as its main components, unsaturated fatty acids and carotenoids. For presenting these characteristics, it is being considered a promising plant for the pharmaceutical and food industry due to its potential application as a herbal and functional food, among others. In this sense, this work aims to present a literature review on araçá and its benefits.
\end{abstract}

Keywords: Araçá red; Yellow araçá; Antioxidants; Application.

\title{
Resumen
}

Los consumidores buscan cada vez más alimentos que tengan compuestos beneficiosos para la salud, también llamados bioactivos o fotoquímicos, que son sustancias derivadas del metabolismo secundario de los vegetales. Brasil tiene una de las mayores biodiversidades del mundo, con fuentes inexploradas de frutos nativos con altos niveles de compuestos bioactivos. Entre estos frutos destaca el araçá (Psidium cattleianum Sabine). Araçá, además de su alto valor nutricional, tiene cantidades importantes de compuestos fenólicos, con epicatequina y ácido gálico como componentes principales, ácidos grasos insaturados y carotenoides. Por presentar estas características, está siendo considerada una planta promisoria para la industria farmacéutica y alimentaria debido a su potencial aplicación como alimento herbario y funcional, entre otros. En este sentido, este trabajo tiene como objetivo presentar una revisión de la literatura sobre araçá y sus beneficios.

Palabras clave: Guacamayo rojo; Guacamayo amarillo; Antioxidantes; Aplicación.

\section{Introdução}

A flora brasileira possui mais de 40.000 espécies vegetais ricas em fitoquímicos. Frutos fontes de antioxidantes fenólicos, flavonóides, polissacarídeos, vitamina $\mathrm{C}$ e os terpenos, com alto potencial para produzir conhecimento e produtos com valor agregado útil em fitomedicina e suplementos alimentares para prevenir e tratar diversos tipos de doenças (Carvalho e Conte-Junior, 2021). Dentre as espécies nativas brasileiras, destaca-se o araçá (Psidium cattleyanum), pertencentes à família Myrtaceae. Esta é uma planta endêmica do Brasil, popularmente conhecido como araçá-vermelho ou araçá-amarelo, além de receber também nomes como araçá-rosa; araçá do mato e araçá do campo (Lopes e Silva, 2018).

O araçá possui alto potencial agroalimentar pela abundância em compostos bioativos, elevados teores de substâncias fenólicas que são positivamente correlacionados com a atividade antioxidante, anti-inflamatória, antimicrobiana, os quais são benéficos a saúde na prevenção de doenças (Possa, 2016; Pereira et al., 2018; Rosário et al., 2020; Meregalli et al., 2020).

Os compostos bioativos que se destacam com ação antioxidante são os compostos fenólicos (antocianinas, flavonoides e ácidos orgânicos) e os carotenoides (licopeno, $\beta$-caroteno, zeaxantina, entre outros) (Rosário et al., 2020). Em comparação com morangos e uvas, o araçá possui teores de compostos fenólicos totais superiores, e na mesma faixa da pitanga e da ameixa. Os teores destes compostos podem variar em função de fatores climáticos, como incidência de sol, solo e grau de maturação (Melo, 2019).

Estudos confirmaram que a prevenção de doenças degenerativas está associada ao consumo de frutas que indicam elevada capacidade antioxidante, e ainda, reforçam que os mesmos são de grande interesse pela capacidade de absorver radicais livres e assim impossibilitar reações oxidativas (Verruck et al., 2018; Bombana et al., 2021). A adição de extrato de araçá em dietas de alta palatabilidade, indicaram que efeitos antihiperglicêmicos e antihiperlipidêmicos, além de propriedades neuroprotetoras (Oliveira et al., 2018).

Em frutas os compostos bioativos são naturais e, portanto, mais aceitáveis para os consumidores (Verruck et al., 2018). Por outro lado, os antioxidantes sintéticos, ainda usados em maioria entre as indústrias, podem se tornar carcinogênicos 
se utilizados em doses inadequadas (Lira et al., 2021). Cada vez é maior o interesse por estudos de descoberta e identificação de compostos naturais, com o objetivo de encontrar novas e promissoras fontes de antioxidantes para a saúde humana.

Com base no exposto, observa-se que o araçá é uma fruta que apresenta elevado valor nutricional assim como muitos frutos disponíveis no mercado, e rica em propriedades bioativas, porém ainda pouca explorada economicamente. Apenas o óleo essencial das folhas do araçá-vermelho é comercializado no Brasil para fins aromaterapêuticos pelo Grupo Laszlo@ (Emporio Laszlo, 2021). Desta forma, considerando as características nutricionais e funcionais do araçá (Psidium cattleianum Sabine), bem como sua distribuição em todo território brasileiro, especialmente na região sul, este trabalho tem como objetivo apresentar uma revisão bibliográfica sobre a fruta e seus benefícios.

\section{Metodologia}

Este trabalho de revisão narrativa (UNESP, 2015) foi desenvolvido por pesquisa bibliográfica realizada em artigos científicos, dissertações e teses oriundas de bases de dados e portais de pesquisa Wiley Online Library, Science Direct, Scielo (Scientific Eletronic Library online). Os critérios de inclusão foram trabalhos publicados de 2000 a 2021 e analisados de junho a dezembro de 2021, de disponibilidade na íntegra e gratuitos, escritos em português e inglês, que abordavam a temática do presente estudo. Ainda para a seleção dos trabalhos as palavras de busca foram "Araçá", "Araçá-vermelho", "Araçáamarelo, "Psidium cattleyanum", "Psidium cattleyanum Sabine", "Extrato de araçá", "Propriedade bioativas", "Composição química”, além disso empregaram-se os operadores logísticos booleanos (AND/OR). Foram excluídas publicações que não respondiam a problemática desta pesquisa. A Figura 1 apresenta o fluxograma de seleção de artigos pesquisados para a realização da revisão bibliográfica.

Figura 1 - Fluxograma de seleção dos artigos para a revisão bibliográfica.

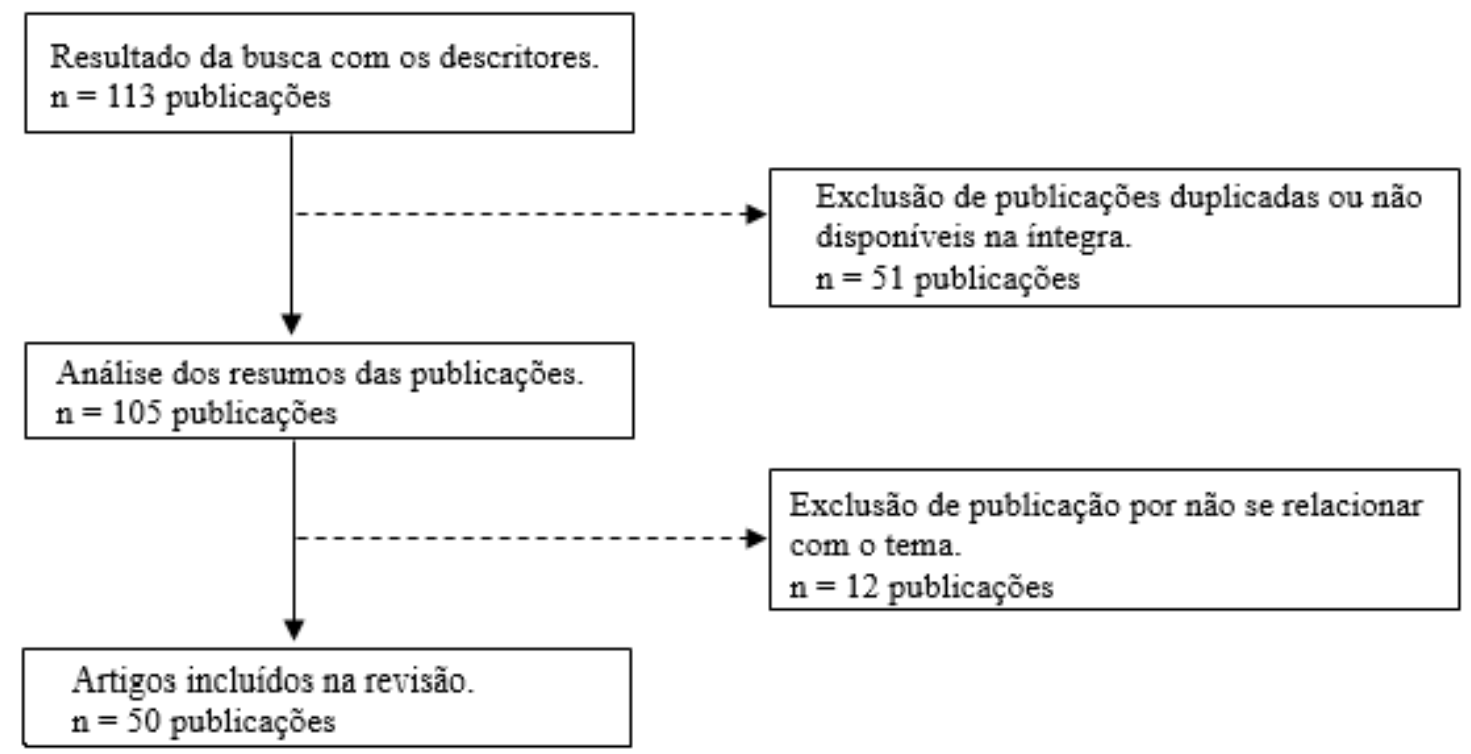

Fonte: Autores.

\section{Desenvolvimento}

\subsection{Araçá}

$\mathrm{O}$ araçá (Psidium cattleyanum) é originário do Sul do Brasil e está distribuído amplamente na região sul e sudeste do Brasil, bem como em outros países da América do Sul (Frazon et al., 2009; Koch, Fachinetto e Bianchi, 2021). O nome "araçá" 
origina-se do tupi ara'sa ou da guarani ara (que significa céu) e aza (olho), ou seja, "fruto com olhos" (Silva Júnior, 2005). Sua classificação taxonômica é: classe Magnoliopsida, ordem Myrtales, família Myrtaceae, gênero Psidium e espécie $P$. cattleyanum (Lopes e Silva, 2018). Do gênero Psidium, são mais de 100 espécies são encontradas no Brasil, destes 5 no Rio Grande do sul: Psidium austrele Cambesèdes; P. incanum (Berg) Burret; P. Luridum (Sprengel) Burret; P. pudifolium Burret $\mathrm{e}$ P. Cattleyanum Sabine (Brasil, 2015)

O melhoramento seletivo para plantas mais produtivas e com baixa vulnerabilidade a pragas e doenças, aliadas a produtos ricos em nutrientes, resultaram na seleção de genótipos no Banco de Germoplasma da Embrapa Clima Temperado, com propagação de duas cultivares, denominadas "Ya-Cy" e "Irapuã". As cultivares Ya-Cy produzem frutos amarelos, que começam a aparecer 1 ano após o plantio e produtividade de até $4 \mathrm{~kg} / \mathrm{planta}$ ao ano. A Irapuã produz frutos com uma cor vermelho púrpura e de um médio a grande porte, com produção que se inicia 2 anos após o plantio, atingindo de 3,4 a 14 kg/planta por ano (Frazon et al., 2009; Lopes e Silva, 2018).

O araçazeiro é uma espécie arbórea, com altura de 3 a $6 \mathrm{~m}$ e tronco com diâmetro de 15 a $25 \mathrm{~cm}$. Suas folhas são simples, coriáceas, glabras. As flores são de coloração branca. Floresce entre os meses de outubro e novembro e a segunda em dezembro. Podendo casualmente florescer em março, sendo assim, os frutos podem ser colhidos de outubro a março (Brasil, 2015; Possa, 2016; Rosário et al., 2020; Meregalli et al., 2020; Pereira et al., 2018). Na região sudeste do Paraná, Brasil, Kosera Neto et al. (2020), relataram que o crescimento do araçá-vermelho ocorre entre julho e novembro, com floração entre outubro e novembro, e amadurecimento dos frutos no final de dezembro a março. Já o crescimento vegetativo do araçáamarelo ocorre entre julho e outubro e entre janeiro e abril, com floração de setembro a novembro e janeiro a março com maturação dos frutos entre dezembro e fevereiro e, de abril a setembro.

$\mathrm{O}$ araçá caracteriza-se por ser um fruto climatérico, de 1,5 a $5 \mathrm{~cm}$ de diâmetro, rico em pectina, com polpa brancaamarelada ou avermelhada suculenta, levemente ácida e várias sementes, que variam de 16 a 100 unidades por fruto (Brasil, 2015). Os frutos são bagas globosas, arredondados, de coloração amarela ou vermelha, de acordo com a espécie (Figura 2).

Figura 2 - Frutos do araçá (P. cattleyanum)

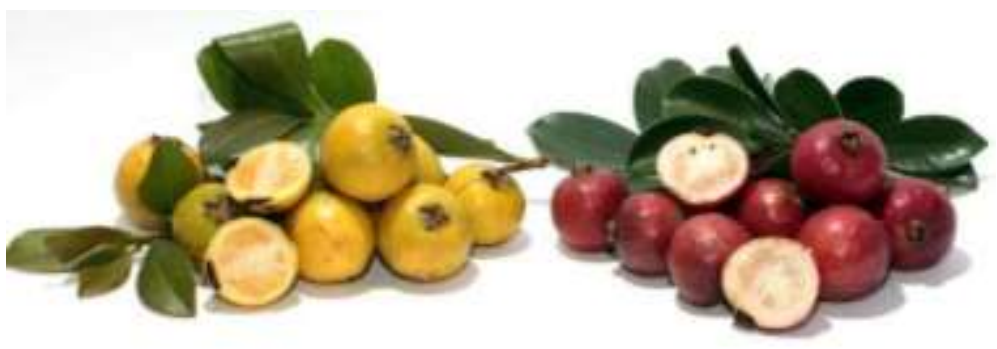

Fonte: Lanzetta (2012).

O araçá apresenta grande potencial econômico em razão da sua composição físico-química e propriedades bioativas relacionadas, na sua maioria, aos compostos fenólicos e ácidos ascórbico, que apresentam uma alta capacidade antioxidante (Melo et al., 2020). É normalmente consumido in natura ou processado em sucos, geleias, doces, sorvetes, entre outros, sua composição química e suas características nutricionais conferem um potencial para industrialização, e de suas sementes pode ser extraído óleo, o qual é utilizado na produção de cosméticos (Zandoná et al., 2020).

\subsection{Caracterização e composição nutricional do araçá}

$\mathrm{O}$ araçá é uma fruta pequena e redonda; quando madura, a casca pode ser vermelha, amarela ou verde, de acordo com a espécie. A parte comestível da fruta possui sabor ácido, mucilaginoso e aromático (Haminiuk et al., 2006; Pereira et al, 
2020). O primeiro estudo sobre o perfil volátil de araçá foi realizado no Japão, em 1980 (Egea et al., 2014). Biegelmeyer et al. (2011), demonstraram que entre araçá-vermelho e amarelo, o principal sesquiterpeno do óleo é o $\beta$-cariofileno, 22,5 e 28,7\%, respectivamente.

Comparado com a maçã, fruta mais consumida no mundo, o araçá apresenta menor valor de calorias em carboidratos e maior quantidade de lipídios e fibras alimentares (Pereira et al., 2020). O araçá possui valor energético de 62Kcal/100g. A sua composição centesimal é de 14,3g de carboidratos, 5,2g de fibras alimentares, 0,6g de lipídeos e 1,5g de proteínas (Brasil, 2015). Valores de proteína entre 1,45 a 1,87g/100g foram relatados por Lopes e Silva (2018). E embora o araçá possa fornecer pequena quantidade de proteínas, proporciona quantidades relevantes de leucina ( $\approx 40 \mathrm{mg} / 100 \mathrm{~g}$ ) (Pereira et al., 2020).

As sementes de araçá oferecem altos níveis de ácidos graxos poliinsaturados e presença de ácidos graxos monoinsaturados. Dietas ricas nesses ácidos estão associadas a um risco reduzido de doenças cardiovasculares e aterogênese (Biegelmeyer et al., 2011, Pereira et al., 2020). O principal ácido graxo em óleo essencial de sementes de araçá-vermelho e amarelo é o ácido linoleico (61,01\%,75,42\%, respectivamente), 10,83\% de ácido oleico de para araçá-vermelho e 14,99\% para o óleo essencial extraído de sementes da fruta amarela (Biegelmeyer et al., 2011).

A polpa de araçá apresenta quantidade total de pectina de 0,72g/100g, com solubilidade de 69,4g/100g (Damiani et al., 2011). No processo de amadurecimento as enzimas poligalacturonase e pectinametilesterase agem degradando a pectina, tornando-a mais solúvel e resultando em um fruto macio (Lopes e Silva, 2018). Da Costa Amaral et al. (2021), relataram que as pectinas do araçá são formadas por longos blocos de ácido galacturônico, parcialmente metilados (DM = 55,9 mol\%) e blocos acetilados, e ramnogalacturonana tipo I com cadeias laterais contendo principalmente arabinose e galactose. Os autores testaram a capacidade de gelificação e o comportamento reológico de uma das amostras, obtendo a gel homogêneo estável.

Apresenta em sua constituição cálcio $(48 \mathrm{mg} / 100 \mathrm{~g})$, ferro $(6,3 \mathrm{mg} / 100 \mathrm{~g})$, potássio $(33 \mathrm{mg} / 100 \mathrm{~g})$. A fruta pode apresentar entre quatro a sete vezes mais vitamina C que frutas cítricas (Brasil, 2015). Melo, Cavalcante e Amante (2020), identificaram que, em amostras de pós liofilizados de araçá vermelho, utilizando a MEV-EDS, os elementos majoritários encontrados foram potássio, magnésio, sódio e cloro sendo iguais para as safras de 2017 e 2018.

\subsection{Compostos bioativas do araçá}

$\mathrm{O}$ araçá ( $P$. cattleianum) é popularmente conhecido por sua ação hipoglicemiante, antibacteriana, antiinfamatória e atividade no sistema nervoso central. O seu consumo, e de frutas em geral, se relaciona com redução de doenças crônicas devido a presença de compostos bioativos (Brasil, 2015; Van't Veer et al., 2000; Oliveira et al., 2018; Pereira et al., 2020). Esses compostos são substâncias derivadas do metabolismo secundário dos vegetais, como antocianinas, flavonóides e ácidos fenólicos. Sua ação antioxidante minimiza danos oxidativos causado por espécies reativas de oxigênio (ROS) (Silva et al., 2014; Das, Goud e Das, 2017). O araçá é associado a fitoquímicos como a epicatequina, ácido gálico, apigenina, isoquercitrina e outros (Medina et al., 2011; Pereira et al, 2020).

\subsubsection{Capacidade antioxidante do araçá}

Os antioxidantes são definidos como qualquer molécula que retarda, previne ou retira um dano oxidativo de uma molécula alvo. Podem ainda preservar as biomoléculas evitando a formação dos radicais livres, impossibilitando a oxidação do substrato, detendo os radicais livres gerados pelo metabolismo através da ação enzimática ou pelos agentes de sacrifício, o qual tem a função de proteger essas biomoléculas (Lira et al., 2021)

As reações de oxidação (ROS) podem produzir radicais livres que se referem a um átomo ou molécula instável/altamente reativo com único elétron, que podem retirar o elétron ou átomo de outros substratos e induzem a decomposição oxidativa do mesmo. Quando produzidos em excesso os radicais livres causam danos às células e tecidos. O 
estresse oxidativo (ROS) está relacionado a doenças como câncer, doenças neurodegenerativas (Alzheimer) e cardiovasculares, diabetes, depressão, e doenças do envelhecimento (Luo et al., 2019). No estresse oxidativo ocorre um desequilíbrio entre o oxidante e os antioxidantes, então ocasionando o dano molecular, e consequentemente em doenças crônicas ou degenerativas, isso pode ser gerado endogenamente ou exogenamente (Sies, 2020).

Os valores da atividade antioxidante em estudo realizado por Pereira et al. (2021), variaram de 154,9 a 330,3 $\mu \mathrm{g} / \mathrm{g}$ nas sementes e 1097,7 a 1277,0 $\mu \mathrm{g} / \mathrm{g}$ na polpa. O ensaio de atividade antioxidante usando o radical estável DPPH é amplamente utilizado para avaliar frutas nativas. $\mathrm{O}$ araçá apresentou maior atividade antioxidante que uvaia ou guabiroba, com valor de $389,7 \mu \mathrm{g} / \mathrm{g}$, semelhante aos resultados observados para sementes.

Meregalli et al. (2020), obtiveram a eficiência da atividade antioxidante, avaliada em função do percentual de inibição de DPPH dos extratos obtidos pelo método convencional de extração por maceração e assistida por ultrassom, resultando em 85,57 e $86,31 \%$ de inibição, respectivamente, utilizando extrato na concentração de $50 \mathrm{mg} / \mathrm{mL}$. Esta eficiência pode ser correlacionada com o alto teor de antocianinas totais da casca do araçá-vermelho. O araçá também apresentou boa proteção antioxidante da levedura (Saccharomyces cerevisiae) contra peróxido de hidrogênio em uma concentração de $25 \%$, resultando em taxas de sobrevivência da levedura acima de 80 \% (Medina et al., 2011).

\subsubsection{Compostos fenólicos do araçá}

Os compostos fenólicos têm grande diversidade química, desempenhando um papel muito importante na saúde humana, pelo seu potencial nutricional, antioxidante, antimicrobiano e antinflamatório (Tuler et al., 2018). Estes compostos são provenientes prioritariamente do metabolismo secundário das plantas, que estão diretamente ligados ao metabolismo primário e sintetizados através da via do acetato e chiquimato e pela metabolização dos fenilpropanoides em plantas (Figura 3).

Figura 3 - Esquema simplificado da rota de produção de compostos fenólicos.

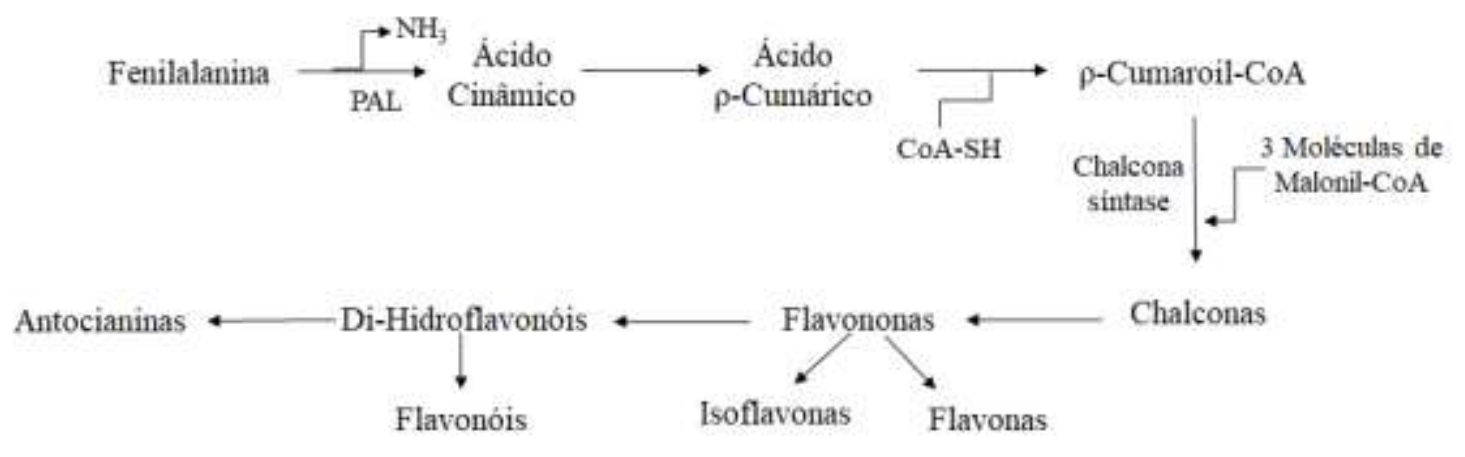

Fonte: adaptado de Taiz e Zeiger (2009).

Os fenilpropanoides, os flavonoides e os taninos são compostos fenólicos que mais se sobressaem em alimentos, sendo fundamental a quantificação e identificação desses devido suas propriedades biológicas e químicas benéficas à saúde. Estão presentes nas frutas, verduras e legumes são as mais importantes fontes de antioxidantes da dieta junto com os carotenoides; ambos apontam a capacidade de proteger o organismo contra danos oxidativos (Melo, 2019).

O consumo de compostos fenólicos traz benefícios à saúde e por esse motivo estão intensificando as pesquisas para identificar, quantificar e avaliar as propriedades antioxidantes dessa classe de moléculas. Entre os mais de 10.000 compostos fenólicos identificados nas plantas, 500 foram encontrados nos alimentos. Neste estudo um total de 43 e 45 compostos fenólicos foram identificados na fração não extraível (NEPC) do araçá-amarelo e vermelho, respectivamente (Mallmann et al., 2020). Diferentes compostos fenólicos têm sido relatados no araçá, como os ácidos elágico, $\rho$-cumárico, clorogênico e gálico 
(Medina et al., 2011; Ribeiro et al., 2014), flavonóides como quercetina, miricetina e epicatequina (Medina et al., 2011) e antocianinas, como cianidina-3-o-glicosídeo e malvidina-3-o-glicosídeo (Dalla Nora et al., 2014).

Mallmann et al. (2020), reforça a relevância da NEPC para os compostos fenólicos totais das plantas. No araçá, essa fração representa aproximadamente $33 \%$ do total de compostos fenólicos do fruto que se ligam à parede celular vegetal e, normalmente, não são explorados. Meregalli et al. (2020), obtiveram teor de compostos fenólicos totais a partir da extração convencional de 477,53 Eq. mg de ácido gálico/100 g de casca, enquanto que para a extração assistida por ultrassom esse parâmetro atingiu 589,49 Eq. mg de ácido gálico/100 g de casca. Diferentes genótipos e diferentes partes da fruta araçá foram estudados por Pereira et al. (2021), sendo que o conteúdo fenólico total foi semelhante entre todos os genótipos avaliados. No entanto, maior teor foi observado nas frações polpa-casca do que nas sementes entre os genótipos. Os valores para este parâmetro variaram de 1418,5 a 1533,4 mg/100g em sementes, e 1933,3 a 2088,5 mg/100g em casca de polpa. A Tabela 1 apresenta os principais compostos fenólicos encontrados no extrato do araçá-vermelho.

Tabela 1 - Compostos fenólicos presentes no extrato de araçá-vermelho.

\begin{tabular}{|c|c|c|c|}
\hline \multirow{2}{*}{ Compostos fenólicos } & \multicolumn{3}{|c|}{ Araçá vermelho } \\
\hline & Fruta & Polpa & Casca \\
\hline \multirow{8}{*}{ Fenólicos totais } & $1,93-2,9 \mu \mathrm{g} / \mathrm{g}$ de fruta & $12,821 \mu \mathrm{g} / \mathrm{g}$ extrato & $7,098 \mu \mathrm{g} / \mathrm{g}$ extrato \\
\hline & (Pereira et al., 2020) & (Ribeiro et al., 2014) & (Ribeiro et al., 2014) \\
\hline & & & 477, 53 Eq.mg de ácido gálico/100 g de casca \\
\hline & - & - & (Extração convencional) \\
\hline & & & (Meregalli et al., 2020) \\
\hline & & & 589,49 Eq.mg de ácido gálico/100 g de casca \\
\hline & - & - & (Ultrassom) \\
\hline & & & (Meregalli et al., 2020) \\
\hline \multirow[t]{2}{*}{ Catequina } & $1,25-1,29 \mu \mathrm{g} / \mathrm{g}$ de fruta & & \\
\hline & (Pereira et al, 2020) & - & - \\
\hline \multirow[t]{2}{*}{ Epicatequina } & $263,9-2130,4 \mu \mathrm{g} / \mathrm{g}$ de fruta & $1,603 \mu \mathrm{g} / \mathrm{g}$ extrato & $885,0 \mu \mathrm{g} / \mathrm{g}$ extrato \\
\hline & (Medina et al., 2011) & (Ribeiro et al., 2014) & (Ribeiro et al., 2014) \\
\hline \multirow[t]{2}{*}{ Ácido Elágico } & $29,1-43,0 \mu \mathrm{g} / \mathrm{g}$ de fruta & $3,818 \mu \mathrm{g} / \mathrm{g}$ extrato & $2,213 \mu \mathrm{g} / \mathrm{g}$ extrato \\
\hline & (Pereira et al., 2020) & (Ribeiro et al., 2014) & (Ribeiro et al., 2014) \\
\hline \multirow[t]{2}{*}{ Ácido Gálico } & & $1510,0 \mu \mathrm{g} / \mathrm{g}$ extrato & $464,0 \mu \mathrm{g} / \mathrm{g}$ extrato \\
\hline & - & (Ribeiro et al., 2014) & (Ribeiro et al., 2014) \\
\hline \multirow[t]{2}{*}{ Quercetina } & $29,1-44,0 \mu \mathrm{g} / \mathrm{g}$ de fruta & $40,5 \mu \mathrm{g} / \mathrm{g}$ extrato & $130,0 \mu \mathrm{g} / \mathrm{g}$ extrato \\
\hline & (Pereira et al., 2020) & (Ribeiro et al., 2014) & (Ribeiro et al., 2014) \\
\hline
\end{tabular}

Fonte: Autores.

Os compostos fenólicos possuem um anel aromático que apresenta um ou mais grupos hidroxila, sua estrutura pode variar desde uma simples molécula fenólica à um complexo polímero de elevado peso molecular. Sendo encontrados na sua forma livre ou ligados a carboidratos, proteínas ou lipídeos (Vuolo et al., 2019). As classes de compostos fenólicos mais importantes encontradas na dieta humana são os ácidos fenólicos, flavonoides e taninos. Quimicamente, os ácidos fenólicos têm no mínimo um anel aromático, onde um hidrogênio pode ser substituído por um grupo hidroxila. São conhecidas mais de 8.000 estruturas de compostos fenólicos (Vuolo et al., 2019). 


\subsubsection{Flavonoides}

Os flavonoides são polifenólicos de baixo peso molecular, produzidos como metabólitos secundários da planta, totalmente difundidos no reino vegetal, representando a maior classe de fenólicos vegetais. São essenciais para a sobrevivência da mesma, atuam na coloração das flores, sinalização, transporte de auxina, inibição proteção ultravioleta e alelopatia (Verruck et al., 2018; Khalid et al., 2019; Owona et al., 2020).

Os flavonoides são substâncias fenólicas que possuem em sua estrutura química anéis aromáticos, tendo como base o 2-fenil-benzopirano (C6-C3- C6). Encontrados em maçã, uva, cebola, repolho, brócolis, chicória, aipo, açaí, cacau, chá e vinho tinto sendo, portanto, constituintes da dieta humana (Araújo, 2020).

Verruck et al. (2018), citam seis grupos de flavonoides, flavonas, flavonóis, chalconas, flavon-3-ol, flavononas e protoantocianidinas, mais estudados em relação à atividade antimicrobiana contra várias espécies de bactérias patogênicas. Segundo Alseekh et al. (2020), já foram identificados mais de 8.000 flavonoides diferentes, e um número considerável é descoberto a cada ano.

Pesquisas sugerem que alguns flavonoides são responsáveis por ação antitumoral considerável, podendo ainda agir como antivirais, anti-hemorrágicos, hormonais, anti-inflamatórios, antimicrobianos, antioxidantes, antialérgicos, na prevenção de doenças cardiovasculares e diabetes tipo 2 (Verruck et al., 2018; Maleki et al., 2019; Mark et al., 2019). Em um estudo feito por Tavsan e Kayali (2019), os flavonoides demostraram efeitos anticâncer nas células cancerosas do ovário. Bakoyiannis et al. (2019), relatam os efeitos dos flavonoides no sistema cognitivo, como aumento da neogênese e função cognitiva apropriada sob condições saudáveis ou patológicas.

\subsubsection{Antocianinas}

As antocianinas são glicosídeos de polihidroxi e polimetoxi derivados de um esqueleto 2-fenil-benzopílico (cátion Flavylium) (Ballistreri et al., 2019; Verma et al., 2020). É um grupo de flavonoides responsáveis pelos pigmentos coloridos presentes nas plantas, conhecidos por suas propriedades físicas, químicas e biológicas. As antocianinas são pigmentos relativamente instáveis, e sua estabilidade é afetada por vários fatores, tais como: $\mathrm{pH}$, temperatura, enzimas, presença de ácido ascórbico, exposição à luz, presença de oxigênio, dióxido de enxofre e íons metálicos (Meregalli et al., 2020).

Algumas indústrias de alimentos, farmacêuticas e cosméticas utilizam as antocianinas como corantes naturais nos processamentos dos alimentos ou por suas funções biológicas as quais atuam como agentes na prevenção de doenças. Podendo ainda, ser empregados como conservantes naturais, e também na preservação de ingredientes alimentares contra estresses ambientais no período de armazenamento e transporte (Belwal et al., 2020; Meregalli et al., 2020).

O consumo das antocianinas traz benefícios à saúde humana, pois reduz o risco de doenças cardiovasculares, diabetes, artrite, doenças da visão e câncer em função das atividades antioxidantes e antinflamatórias (Thornthwaite et al., 2020). Meregalli et al. (2020), obtiveram teores de antocianinas maiores que 100 Eq. mg cianidina-3glicosídeo 100 g casca de araçávermelho, caracterizando a casca desta fruta como uma boa fonte desse composto.

\subsection{Possibilidades de aplicação do araçá e de seus componentes}

$\mathrm{O}$ araçá pode ser consumido in natura ou processado, sob a forma de sucos, sorvetes e chás liofilizados (Pereira et al., 2018). Todavia, por ser um fruto sazonal, não está disponível para o consumo durante todo o ano e apresenta um curto período de armazenamento na forma refrigerada devido a sua alta perecibilidade (Amarante et al., 2009; Pereira et al., 2018). Desenvolvimento de técnicas de desidratação, liofilização e outras se tornam alternativas para a preservação dos frutos, aplicadas tanto aos frutos inteiros ou em partes, objetivando a comercialização na forma de chips de frutas, como misturas na forma de pó para formulações ou extratos de compostos bioativos (Tan et al., 2020; Melo et al., 2020; Meregalli et al., 2020). 
Pesquisas com o araçá e de seus subprodutos, em relação a seus atributos sensoriais e bioativos para benefícios à saúde, vem sendo estudado. Dalla Nora et al. (2014), utilizaram pó liofilizado de araçá em estudo in vivo e demonstraram redução de níveis de glicose, colesterol total e frações nos animais que foram tratados com araçá.

O extrato de araçá mostrou atividade antimicrobiana para Staphylococcus aureus e Listeria monocytoneneses (Pereira et al., 2019) e Staphylococcus aureus (de Lima et al., 2020), indicando potencialidade de utilização do mesmo para controle microbiano em alimentos, substituindo conservantes sintéticos.

Da Costa Amaral et al. (2021), avaliaram a capacidade de gelificação da pectina extraída da polpa do araçá e obtiveram um gel homogêneo e estável, tornando-se assim uma alternativa às pectinas críticas e para a indústria de alimentos empregar na formação de geleias com baixo teor de açúcar, uma vez que não requer a adição de sacarose para gelificação (Noreen et al., 2017).

Recentemente, vem crescendo o interesse por compostos fenólicos de frutas capazes de inibir as enzimas digestivas. Os extratos de araçá foram testados para o controle da hiperglicemia e diminuição dos níveis de glicemia e triglicerídeos (de Souza Cardoso et al., 2018). Estudos demonstram que os extratos de araçá inibem a atividade de $\alpha$-glucosidase (Vinholes et al., 2017; Pereira et al., 2021), $\alpha$-amilase (Pereira et al., 2021) e lipase pancreática (Pereira et al., 2021). A aplicação destes extratos na inibição direcionada das enzimas $\alpha$-glucosidase e $\alpha$-amilase é usada para retardar a absorção intestinal de glicose, reduzindo os níveis de glicose no sangue pós-prandial e a enzima lipase tem papel fundamental no metabolismo lipídico, e sua inibição tem sido estudada como alvo para o tratamento da obesidade.

\section{Considerações Finais}

O araçá é comprovadamente fonte de nutricional e de compostos bioativos, especialmente fenólicos, os quais são relacionados a sua atividade antioxidante. Desta forma, o araçá deve ser recomendado para consumo, tanto in natura quanto processado na forma de sucos, geleias, doces, sorvetes, iogurtes entre outros, bem como o uso dos extratos como corantes e conservantes de alimentos processados, visto que esses contribuem de forma favorável para uma dieta equilibrada auxiliando assim para prevenção de doenças.

\section{Agradecimentos}

Os autores agradecem à Coordenação de Aperfeiçoamento de Pessoal de Nível Superior - Brasil (CAPES) - Código Financeiro 001, Conselho Nacional de Desenvolvimento Científico e Tecnológico - Brasil (CNPq) e Fundação de Amparo à Pesquisa do Estado do Rio Grande do Sul - Brasil (FAPERGS) para bolsas e apoio financeiro.

\section{Referências}

Alseekh, S., Souza P. L., Benina, M., \& Fernie, R. A. (2020). The style and substance of plant flavonoid decoration: towards defining both structure and function. Phytochemistry, 174, 112347.

Amarante, C. V. T., Steffens, C. A., \& Espíndola, B. P. (2009). Preservação da qualidade pós-colheita de araçá-vermelho através do tratamento com 1metilciclopropeno e do acondicionamento em embalagens plásticas sob refrigeração. Rev. Bras. Fruticultura, 31(4), 969-976.

Araújo, L. C. A. (2020). Frutos do bioma cerrado: avaliação da atividade antioxidante in vitro e efeitos in vivo em modelo experimental Caenorhabditis elegans. Tese de Doutorado em Biotecnologia e Biodiversidade, Faculdade de Ciências Biológicas e Ambientais, Universidade Federal da Grande Dourados, Dourados, MS, Brasil.

Bakoyiannis, I., Daskalopoulou, A., Pergialiotis, V., \& Perrea, D. (2019). Phytochemicals and cognitive health: Are flavonoids doing the trick? Biomedicine \& Pharmacotherapy, 109, 1488-1497.

Ballistreri, G., Fabroni, S., Romeo, V. F., Timpanaro, N., Amenta, M., \& Rapisarda, P. (2019). Anthocyanins and Other Polyphenols in Citrus Genus: Biosynthesis, Chemical Profile, and Biological. In Watson, R.R (Ed.). Activity Polyphenols in Plants (chap. 13, pp. 191-215). Amsterdan: Elsevier. 
Belwal, T., Singh, G., Jeandet, P., Pandeye, A., Girib, L., Ramolaf, S., Bhattb, D. I., Venskutonisg, R. P., Georgievh, I. M., Clementd, C., \& Luoa, Z. (2020). Anthocyanins, multi-functional natural products of industrial relevance: Recent biotechnological advances. Biotechnology Advances, 1 (43), 107600.

Biegelmeyer, R., Andrade, J. M., Aboy, A. L., Apel, M. A., Dresch, R.R., Marin, R., Raseira, M.C., Henriques, A. T. (2011). Comparative analysis of the chemical composition and antioxidant activity of red (Psidium cattleianum) and yellow (Psidium cattleianum var. lucidum) strawberry guava fruit. Journal of Food Scince, 76 (7), C991-C996.

Bombana, V. B., Oro, G.E.D., Rigo, D., Polina, C. C., Denti, A. F., Tres, B. P., Wisniewski, M. S. W., Steffens, J., Paroul, N., Dallago, R. M., Backes, G. T., Cansian, R. L. (2021). Influence of drying on bioactive compounds and antioxidant activity of fruits of guabiju (Myrcianthes pungens). Research, Society and Development, v. 10, n. 8, e5510817024, 2021

Brasil. Ministério da Saúde. Secretaria de Atenção à Saúde. Departamento de Atenção Básica. Alimentos regionais brasileiros/Ministério da Saúde, Secretaria de Atenção à Saúde, Departamento de Atenção Básica. 2. Ed. Brasília: Ministério da Saúde, 2015.

Carvalho, A.P.A., \& Conte-Junior, C.A. (2021). Health benefits of phytochemicals from Brazilian native foods and plants: Antioxidant, antimicrobial, anticancer, and risk factors of metabolic/endocrine disorders control. Trends in Food Science \& Technology, 111, 534-548.

Costa Amaral, S., Roux, D., Caton, F., Rinaudo, M., Barbieri, S. F., Silveira, J. L. M. (2021). Extraction, characterization and gelling ability of pectins from Araçá (Psidium cattleianum Sabine) fruits. Food Hydrocolloids, 121, 106845.

Dalla Nora, C., Danelli, D., Souza, L. F., Rios, A. D. O., Jong, E. V. De, \& Flôres, S. H. (2014). Protective effect of guabiju (Myrcianthes pungens (O. Berg) D. Legrand) and red guava (Psidium cattleyanum Sabine) against cisplatin-induced hypercholesterolemia in rats. Brazilian Journal of Pharmaceutical Sciences, $50,483-491$.

Damiani, C., Vilas Boas, E. V. B., Asquieri, E. R., Lage, M. E., de Oliveira, R. A., da Silva, F. A., Pinto, D. M., Rodrigues, L. J., da Silva, E. P., \& de Paula, N.R.F. (2011). Characterization of fruits from the savanna: Araça (Psidium guinnensis Sw.) and Marolo (Annona crassiflora Mart.). Ciência e Tecnologia de Alimentos, 31(3), 723-729.

Das, A. B., Goud, V. V., \& Das, C. (2017). Extraction of phenolic compounds and anthocyanin from black and purple rice bran (Oryza sativa L.) Using ultrasound: A comparative analysis and hytochemical profiling. Ind. Crops Prod. 95, 332-341.

Lima, A. S., Maia, D.V., Haubert, L., Oliveira, T. L., Fiorentini, A. M., Rombaldi, C. V., \& da Silva, W. P. (2020). Action mechanism of araçá (Psidium cattleianum Sabine) hydroalcoholic extract against Staphylococcus aureus. LWT - Food Science and Technology, $119,108884$.

De Souza Cardoso, J.; Oliveira, P. S., Bona, N. P., Vasconcellos, F. A., Baldissarelli, J., Vizzotto, M., Soares, M. S., Ramos, V. P., Spanevello, R. M., Lencina, C. L., Tavares, R. G., \& Stefanello, F. M. (2018). Antioxidant, antihyperglycemic, and antidyslipidemic effects of Brazilian-native fruit extracts in an animal model of insulin resistance. Redox Report, 23 (1), 41-46.

Egea, M. B., Pereira-Netto, A. B., Cacho, J., Ferreira, V., \& Lopez, R. (2014). Comparative analysis of aroma compounds and sensorial features of strawberry and lemon guavas (Psidium cattleianum Sabine). Food Chemistry, 164, 272-277.

Emporio Laszlo. (2021). https://www.emporiolaszlo.com.br/oleo-essencial-de-araca-rosa.html.

Frazon, R. C., Campos, L. Z. O., Proença, C. E. B., \& Silva, J. C. S. (2009). Araçás do gênero Psidium: espécies, ocorrências, descrição e usos. Documentos Embrapa Cerrados: Brasília. 48 p.

Haminiuk, C. W. I., Sierakowski, M. R., Vidal, J. R. M. B., \& Masson, M. L. (2006). Influence of temperature on the rheological behavior of whole araçá pulp (Psidium cattleianum sabine). LWT - Food Science and Technology, 39, 427-431.

Khalid, M., Rahman, S., Bilal, M., \& Dan-Feng, H. (2019). Role of flavonoids in plant interactions with the environment and against human pathogens-A review. Journal of Integrative Agriculture, 18 (1), 211-230.

Koch, S., Fachinetto, J. M., \& Bianchi, V. (2021) Distribuição geográfica potencial atual e futura de Araçá (Psidium Catlleianum) para a América do Sul. Brazilian Journal of Development, 7 (2), 16268-16277.

Kosera Neto, C., Radaelli, J. C., Guollo, K., Wagner Júnior, A., Zanela, J., \& Frazon, R. C. (2020). Acompanhamento fenológico do morango-goiaba na Região Sudeste do Paraná. Pesquisa, Sociedade e Desenvolvimento, 9 (10), e8319109180.

Lanzetta, P. (2012). Foto de Araçá Unidade: Embrapa Clima Temperado. Recuperado em 20 dezembro, 2021, de https://www.embrapa.br/busca-de-imagens//midia/640002/araca.

Lira, L. A., Vesoloski, F. J., Peruzzolo, M., Flôres, Z. D., Cansian, R. L., \& Paroul, N. (2021). Atividades Antioxidante, Antimicrobiana e Compostos Fenólicos de Extratos Comercial e In Natura de Cúrcuma Longa. Perspectiva, 45 (169), 107-114.

Lopes, M. M. A., \& Silva. E. O. (2018). Araçá - Psidium cattleyanum Sabine. Exotic Fruits - Reference Guide, 31-36.

Luo, J., Mills, K., Le Cessie, S., Noordam, R., \& Van Heemst, D. (2019) Ageing, Age-related Diseases and Oxidative Stress: What to Do Next? Ageing Research Reviews, 57, 100982.

Maleki, S., Crespo, J. F., \& Cabanillas, B. (2019). Anti-inflammatory effects of flavonoids. Food Chemistry, $199,125124$.

Mallmann, L. P., Tischer, B., Vizzotto, M., Rodrigues, E., \& Manfroi, V. (2020). Comprehensive identification and quantification of unexploited phenolic compounds from red and yellow araçá (Psidium cattleianum Sabine) by LC-DAD-ESI-MS/MS, Food Research International, 131, 108978

Mark, R., Lyu, X., Lee, J. J. L., Parra-Saldívar, R., \& Chen, N. W. (2019). Sustainable production of natural phenolics for functional food applications. Journal of Functional Foods, 57, 233-254. 
Medina, A. L., Haas, L. I. R., Chaves, F. C., Salvador, M., Zambiazi, R. C., Da Silva, W. P., Nora, L., \& Rombaldi, C. V. (2011). Araçá (Psidium cattleianum Sabine) fruit extracts with antioxidant and antimicrobial activities and antiproliferative effect on human cancer cells. Food Chemistry, $128(4), 916-922$.

Melo, W. D. (2019). Propriedades físico-químicas e características histoquímicas do araçá vermelho (Psidium catteyanum Sabine). Dissertação de Mestrado em Ciência dos Alimentos. Universidade Federal de Santa Catarina, Florianópolis, SC, Brasil.

Melo, W. D.; Cavalcante, M.D.B., \& Amante E.R. (2020). Characterization of the red araçá (Psidium Cattleianum Sabine) lyophilized in Powder. Brazilian Journal of Development, 6 (5), 29868-29875.

Meregalli, M. M., Puton, S. M. B., Dal'maso, F. C., Amaral, U. A., Zeni, J., Cansian, R. L., Mignoni, M. L., \& Backes, G. T. (2020). Conventional and ultrasound- assisted methods for extraction of bioactive compounds from red araça peel (Psidium cattleianum Sabine). Arabian Journal of Chemistry, 13, 5800-5809.

Noreen, A., Nazlic, Z., Akrama, J., Rasulb, I., Manshaa, N.Y., Iqbald, R., Tabasum, S., Zuber, M., \& Zia, K. M. (2017). Pectins functionalized biomaterials, a new viable approach for biomedical applications: A review. International Journal of Biological Macromolecules, 101, 254-272.

Oliveira, P. S., Chaves, V. C., Soares, M. S. P., Bona, N. P., Mendonça, L. T., Carvalho, F. B., Gutierres, J. M., Vasconcellos, F. A., Vizzotto, M., Vieira, A., Spanevello, R. M., Reginatto, F. H., Lencina, C. L., \& Francieli Moro Stefanello, F. M. (2018). Southern Brazilian native fruit shows neurochemical, metabolic and behavioral benefits in an animal model of metabolic syndrome. Metabolic Brain Disease, 33, 1551-1562.

Owona, A. B., Abia, A. W., \& Moundipa F. P. (2020). Natural compounds flavonoids as modulators of inflammasomes in chronic Diseases. International Immunopharmacology, 84, 106498.

Pereira, E. S., Camargo, T. M., Radunz, M., Ribeiro, J. A., Alvez, P. I. C., Vizzotto, M., \& Gandra, E. A. (2019). Determinação de atividade antimicrobiana do araçá vermelho (Psidium cattleianum L.). In: A produção do Conhecimento nas Ciências Agrárias e Ambientais, 2, $217-226$.

Pereira, E. S., Vinholes, J. R., Camargo, T. M., Raphaelli1 C. O., Ferri N. M. L., Nora, L., \& Vizzotto, M. (2021). Araçá (Psidium cattleianum Sabine): bioactive compounds, antioxidant activity and pancreatic lipase inhibition. Food Technology Ciência Rural, 51 (11), e20200778.

Pereira, E. S., Vinholes, J.R., Franzon, R., Dalmazo, G., Vizzotto, M., \& Nora, L. (2018). Psidium cattleianum fruits: A review on its composition and bioactivity. Food Chemistry, 258, 95-103.

Possa, J. (2016). Compostos Bioativos e Capacidade Antioxidante De Araçás (Psidium Cattleianum Sabine) Morfotipo Amarelo e Vermelho Cultivados No Rio Grande Do Sul. Monografia de Bacharel em Nutrição, Universidade Federal do Rio Grande do Sul, Brasil.

Ribeiro, A.B., Chisté, R.C., Freitas, M., da Silva, A.F., Visentainer, J.V., \& Fernandes, E. (2014). Psidium cattleianum fruit extracts are efficient in vitro scavengers of physiologically relevant reactive oxygen and nitrogen species. Food Chemistry, 165, 140-148.

Rosário, M. F., Biduski, B., Santos, F. D., Hadlish, V. E., Tormen, L., Dos Santos, F. H. G., \& Pinto, Z. V. (2020). Red Araçá pulp microencapsulation by hydrolyzed pinhão Starch, tara qnd qrabic gums. J. Sci. Food Agric., 101 (5), 2052-2062.

Sies, H. (2020). Oxidative eustress and oxidative distress: Introductory remarks. In: Oxidative Stress (Chap. 1, pp. 3-12). Amsterdan: Elsevier.

Silva Junior, M. C. (2005). 100 árvores do cerrado: guia de campo. Brasília, DF: Rede de Sementes do Cerrado, 278p.

UNESP (2015). Faculdade de Ciências Agronômicas. Biblioteca Prof. Paulo de Carvalho Mattos. Tipos de revisão de literatura. Botucatu, 2015. https://www.fca.unesp.br/Home/Biblioteca/tipos-de-evisao-de-literatura.

Taiz, L., \& Zeiger, E. (2009). Fisiologia Vegetal. $4^{\mathrm{a}}$ Ed. Artmed: Porto Alegre, RS, Brasil.

Tavsan, Z; \& Kayali, H. A. (2019). Flavonoids showed anticancer effects on the ovarian cancer cells: Involvement of reactive oxygen species, apoptosis, cell cycle and invasion. Biomedicine \& Pharmacotherapy, 116, 109004.

Thornthwaite, J. T., Seth, P., Thibado, P. S., \& Thornthwaite, A. K. (2020). Bilberry anthocyanins as agents to address oxidative stress Pathology. In: Preedy, V.R. Pathology Oxidative Stress and Dietary Antioxidant. (chap. 17, pp. 179-187). Amsterdan: Elsevier.

Tuler, C. A., Proença, B. E. C., Carrijo, T. T., \& Peixoto, L. A. (2018). Typification and nomenclatural notes on Psidium cattleyanum (Myrtaceae). International Association for Plant Taxonomy (IAPT), 67 (6), 1194-1198.

Van't Veer, P., Janson, M., Klert, M., \& Kok, F. (2000). Fruits and vegetables in the prevention of cancer and cardiovascular disease. Public Health Nutrition, 3, 103-107.

Verma, K., \& Srivastavp, P. (2020). Bioactive compounds of rice (Oryza sativa L.): Review on paradigm and its potential benefit in human health. Trends in Food Science \& Technology, 97, 355-365.

Verruck, S., Prudencio, S. E., \& Da Silveira, M. S. (2018). Compostos Bioativos Com Capacidade Antioxidante e Antimicrobiana em Frutas. Revista do Congresso Sul Brasileiro de Engenharia de Alimentos, 4 (1), 112.

Vinholes, J., Lemos, G., Barbieri, R.L., Franzon, R. C., \& Vizzotto, M. (2017). In vitro assessment of the antihyperglycemic and antioxidant properties of araçá, butiá and pitanga. Food Bioscience, 19, 92-100.

Vuolo, M. M., Lima, V. S., \& Maróstica Junior, R. (2019). Phenolic Compounds: Structure, Classification, and Antioxidant Power. In: Campos, M.R.S. Bioactive Compounds Health Benefits and Potential Applications. (chap. 2, pp. 33-50). Amsterdan: Elsevier.

Zandoná, G. P., Bagatini, L., Woloszyn, N., Cardoso, J. S., Hoffmann, J. F., Moroni, L. S., Stefanello, F. M., Junges, A., \& Rombaldi, C. V. (2020). Extraction and characterization of phytochemical compounds from aracazeiro (Psidium cattleianum) leaf: Putative antioxidant and antimicrobial properties. Food Research International, 137, 109573. 